\title{
$m i R-181 b$ increases drug sensitivity in acute myeloid leukemia via targeting HMGB1 and Mcl-1
}

\author{
FEI LU ${ }^{1}$, JINGRU ZHANG ${ }^{1}$, MIN JI ${ }^{1}$, PENG LI ${ }^{1}$, YAHUI DU ${ }^{1}$, HONGCHUN WANG ${ }^{1}$, \\ SHAOLEI ZANG ${ }^{1}$, DAOXIN MA ${ }^{1}$, XIULIAN SUN ${ }^{2}$ and CHUNYAN JI ${ }^{1}$ \\ ${ }^{1}$ Department of Hematology and ${ }^{2}$ National Key Lab of Otolaryngology, \\ Qilu Hospital, Shandong University, Jinan 250012, P.R. China
}

Received January 29, 2014; Accepted April 4, 2014

DOI: 10.3892/ijo.2014.2390

\begin{abstract}
Multidrug resistance (MDR) remains the major cause of disease relapse and poor prognosis in adults with acute myeloid leukemia (AML). Emerging evidence shows that drug resistance not only exists against conventional chemotherapeutic drugs, but also limits the efficacy of new biological agents. Therefore, it is important to elucidate the mechanisms through which AML patients develop drug resistance. MicroRNAs have been shown to play an important role in regulating the chemotherapy resistance in AML. A detailed understanding of the mechanisms of microRNA that are clinically relevant in AML may enhance our ability to predict and overcome drug resistance. Here, we demonstrated, for the first time, that $m i R-181 b$ was decreased significantly in human multidrug-resistant leukemia cells and relapsed/refractory AML patient samples. Overexpression of $m i R-181 b$ increased the sensitivity of leukemia cells to cytotoxic chemotherapeutic agents and promoted drug-induced apoptosis. Moreover, $m i R-181 b$ inhibited HMGB1 and Mcl-1 expression by direct binding to their 3'-untranslated regions. In addition, HMGB1 was expressed at high levels in relapsed/refractory AML patients and suppression of HMGB1 via RNA interference sensitized multidrug-resistant leukemia cells to chemotherapy and induced apoptosis. In conclusion, these results provide a strong rationale for the development of $m i R-181 b$-based therapeutic strategies for the enhancement of efficacy in AML treatment.
\end{abstract}

\section{Introduction}

Acute myeloid leukemia (AML) is a clonal disorder of hematopoiesis characterized by the uncontrolled proliferation and accumulation of immature and dysfunctional hematopoietic progenitors accompanied by blockage in normal hematopoi-

Correspondence to: Dr Chunyan Ji, Department of Hematology, Qilu Hospital, Shandong University, 107 West Wenhua Road, Jinan 250012, P.R. China

E-mail: jichunyan@sdu.edu.cn

Key words: miRNA, HMGB1, Mcl-1, acute myeloid leukemia esis. During the last decade, chemotherapy has been widely used as a main approach and preferred therapy for AML treatment. However, the majority of adults diagnosed with AML are destined to relapse, and the major cause of relapse and therapeutic failure in AML is resistance to chemotherapy (1). Many factors account for the occurrence of chemotherapeutic multidrug resistance (MDR), including overexpression of drug resistance-related proteins, alterations in drug targets, escape from cell cycle checkpoints, altered pharmacokinetics, increased drug efflux and stem cell development (2). Given the pressing need to improve outcomes in AML patients, it is crucial to elucidate the mechanisms of chemoresistance.

MicroRNAs (miRNAs, miRs) represent a new class of small, non-coding endogenous RNAs that range in size from 19 to 25 nucleotides (nt) and can negatively regulate target gene expression at the post-transcriptional level. Mature miRNAs are incorporated into the RNA-induced silencing complex (RISC) to cause either degradation or inhibition of translation by binding to the 3'-untranslated region (3'-UTR) of target mRNAs $(3,4)$. Due to their diverse functions in cell proliferation, apoptosis, invasion, cell differentiation, cell cycle progression, and hematopoiesis, overwhelming evidence has indicated the important regulatory roles of miRNAs during carcinogenesis and chemoresistance $(5,6)$. Moreover, modulation of these dysregulated miRNAs sensitizes cancer drug-resistant cells to chemotherapy, suggesting the potential of miRNAs as targets for anticancer drug resistance. For example, overexpression of $m i R-331-5 p$ and/or $m i R-27 a$ can effectively increase the drug sensitivity of leukemia DOX-resistant cells. Furthermore, $m i R-331-5 p$ and $m i R-27 a$ were verified to target the multidrug resistance 1 gene (MDR1), the most extensively studied gene directly involved in drug resistance (7).

$m i R-181 b$ belongs to the $m i R-181$ family, which is known to be evolutionarily conserved among the vertebrate lineage, with high homology (8). Functional research identified $m i R-181 b$ as a key regulator of restricting B cell lymphomagenesis. miR-181b impairs the class switch recombination (CSR) reaction and results in the downregulation of activationinduced cytidine deaminase (AID) in activated B cells (9). It is important to note that the effects of $m i R-181 b$ in chemoresistance vary according to different tumor microenvironments. In hepatocellular carcinoma (HCC) cells, $m i R-181 b$ enhances resistance to the anticancer drug doxorubicin (10). A markedly 
enhanced expression of $m i R-181 b$ was also shown in more aggressive breast cancers and chemotherapy-resistant breast cancer cells, and knockdown of $m i R-181 \mathrm{~b}$ can be used to render breast tumors more responsive to tamoxifen $(11,12)$. In contrast, forced $m i R-181 \mathrm{~b}$ expression sensitizes human MDR gastric cancer cells and lung cancer cells to chemotherapyinduced apoptosis by directly targeting Bcl-2 protein (13). Also, miR-181b is downregulated in chronic lymphocytic leukemia (CLL) compared to normal controls (14-16), and its expression further decreases during the progression of CLL; indeed, downregulation of $m i R-181 a$ and $m i R-181 b$ was associated with shorter overall survival (OS) and disease-free survival in CLL patients (17). Nevertheless, whether $m i R-181 b$ is mechanistically associated with AML progression and relapse remains unknown.

$m i R-451$ is a positive regulator of late-stage maturation of committed erythroid precursors (18). Many studies have shown that $m i R-451$ is widely dysregulated in human cancers and plays a critical role in tumorigenesis and tumor progression $(19,20)$. In addition, $m i R-451$ is involved in mediating the resistance of breast cancer cells to the chemotherapeutic drug doxorubicin through regulating MDR1 expression (21). Aberrant expression of $m i R-486-5 p$ is a frequent molecular event that has important functions in human malignances $(22,23)$; however, evidence of biological roles for $m i R-451$ and $m i R-486$ in relapsed/refractory AML has not yet been reported.

Therefore, based on the important functions of miRNAs in AML initiation and progression, we sought to investigate roles of these 3 miRNAs in chemoresistance of AML. We provided evidence that overexpression of $m i R-181 b$ increased the drug sensitivity of AML MDR cells by targeting high-mobility group box-1 protein (HMGB1) and myeloid cell leukemia-1 (Mcl-1). In conclusion, the identification of $m i R-181 b$ function highlights a new approach for the development of drug resistance therapy in AML.

\section{Materials and methods}

Patient samples. Forty-three AML patients, including 31 newly diagnosed AML patients who had not undergone any therapy or treatment and 12 relapsed/refractory AML patients, were included in our study. All relapsed/refractory AML cases either failed to respond to initial chemotherapy or relapsed after initial complete remission (CR). We excluded patients with inherently resistant AML in the newly diagnosed AML group. Informed consent was obtained from all patients in accordance with the Declaration of Helsinki and with approval of the Medical Ethics Committee of Qilu Hospital, Shandong University. Mononuclear cells (MNCs) from bone marrow aspirates were isolated by density-gradient centrifugation with the use of Ficoll-Paque Plus (Ficoll, Pharmacia LKB Biotechnology, Piscataway, NY, USA). Among those AML patients, 3 matched-pair BM samples were available both at the diagnosis time prior to treatment and the relapsed/refractory state. Detailed clinical information for the AML patients is summarized in Table I.

Cell culture and transfection. The human leukemia cell lines K562 and HL-60 and their multidrug-resistant counterparts, K562/A02 and HL-60/ADM, were purchased from the
Institute of Hematology and Blood Diseases Hospital, Chinese Academy of Medical Sciences and Peking Union Medical College (Tianjin, China). Cells were cultured in complete medium (RPMI-1640 supplemented with $10 \%$ fetal bovine serum, $100 \mathrm{U} / \mathrm{ml}$ penicillin $\mathrm{G}, 100 \mu \mathrm{g} / \mathrm{ml}$ streptomycin, and $2 \mathrm{mM} \mathrm{L}$-glutamine), at $37^{\circ} \mathrm{C}$ in humidified air containing $5 \% \mathrm{CO}_{2}$ and were routinely subcultured every 2-3 days. In addition, in order to maintain the MDR phenotype, doxorubicin was added to the medium of drug-resistant cell lines until 2 weeks before use in experiments.

The synthetic $m i R$ - $181 \mathrm{~b}$ mimic, $m i R$ - $181 b$ inhibitor, and negative controls were purchased from GenePharma (Shanghai, China). Short hairpin RNA targeting human HMGB1 was synthesized from Ribobio (Guangzhou, China). Transfection of miRNAs and short hairpin RNAs was performed with Lipofectamine 2000 reagent (Invitrogen, Carlsbad, CA, USA) in accordance with the manufacturer's protocol.

Cell viability assay. Cells were seeded in 96-well culture plates at density of $5 \times 10^{4}$ cells $/ \mathrm{ml}$ and were treated with serial dilutions of doxorubicin (DOX) or cytarabine (Ara-C) for $48 \mathrm{~h}$. Ten microliters of CCK8 (Beyotime, China) was added to each well, and the cells were incubated at $37^{\circ} \mathrm{C}$ for $4 \mathrm{~h}$. The absorbance in each well was read at $450 \mathrm{~nm}$ by an automated microplate spectrophotometer (Thermo Scientific, USA), with a reference wavelength of $650 \mathrm{~nm}$. Each sample was measured in triplicate, and experiments were repeated 3 times.

Apoptosis assay. Apoptosis was detected using an Annexin V/ FITC and PI apoptosis detection kit (Invitrogen). Briefly, after treatment with DOX $(2.0 \mu \mathrm{g} / \mathrm{ml})$ or Ara-C $(1.0 \mu \mathrm{M})$ for $48 \mathrm{~h}$, $2 \times 10^{5}$ cells were harvested, resuspended in $100 \mu \mathrm{l}$ flow cytometry binding buffer, and stained with $5 \mu 1$ Annexin V/FITC followed by $1 \mu \mathrm{l}$ PI. Cells were then incubated in the dark for $15 \mathrm{~min}$ at room temperature, and $400 \mu \mathrm{l}$ binding buffer was added. The cells were immediately measured by FACSCalibur (Becton-Dickinson, CA, USA).

Quantitative real-time polymerase chain reaction ( $q R T-P C R$ ) analysis. Total RNA was extracted from all samples using TRIzol reagent (Invitrogen). To detect $m i R-181 b$ expression, cDNA was reverse transcribed from total RNA using special stem-loop primers and the mirVana reverse transcription kit (Ambion Inc., Austin, TX, USA), followed by qPCR using TaqMan primer/probe sets from Ambion. U6 small nucleolar RNA was used as an internal control for miRNAs. To detect Mcl-1 and HMGB1 expression, cDNA was synthesized from $\sim 1 \mu \mathrm{g}$ total RNA using the M-MLV RTase cDNA Synthesis kit (Takara, Dalian, China) according to the manufacturer's instructions. Quantitative PCR was conducted on an Applied Biosystems 7900HT system (ABI Prism, Foster City, CA, USA) with SYBR Green PCR Master Mix (Toyobo, Osaka, Japan). GAPDH was used to normalize Mcl-1 and HMGB1 expression levels. Each sample was measured in triplicate, and fold-changes in mRNA expression levels were calculated using the comparative threshold cycle $(\mathrm{Ct})$ method. The sequences of primer pairs specific for each gene are shown in Table II.

Western blot analysis. Cells were lysed with RIPA buffer (0.15 mM NaCl, $0.05 \mathrm{mM}$ Tris-HCl, $\mathrm{pH} \mathrm{7.5,} \mathrm{1 \%} \mathrm{Triton,}$ 
Table I. Characteristics of the 43 AML patients.

\begin{tabular}{llc}
\hline Variables & Newly diagnosed & Relapsed/refractory \\
\hline No. of patients & 31 & 12 \\
Gender (male/female) & $14 / 17$ & $6 / 6$ \\
Age: median years (range) & $45(14-80)$ & $47.5(13-78)$ \\
WBC count $\left(10^{9} /\right.$ l) median: (range) & $13.67(1.28-259.8)$ & $7.55(1.09-204.3)$ \\
Hemoglobin $(g / d)$ median: (range) & $78(43-125)$ & $84(68-157)$ \\
Platelet count $\left(10^{9} /\right.$ l) median: (range) & $40(3-250)$ & $116.5(11-236)$ \\
FAB classification & & \\
No. of patients & & 0 \\
M0 & 1 & 0 \\
M1 & 2 & 2 \\
M2 & 5 & 3 \\
M3 & 5 & 2 \\
M4 & 8 & 5 \\
M5 & 10 & 0 \\
M6 & 0 & \\
\hline
\end{tabular}

FAB, French-American-British classification; WBC, white blood cells.

Table II. Primer sets and genes included in qPCR.

\begin{tabular}{llc}
\hline Name & \multicolumn{1}{c}{ Forward primer } & Reverse primer \\
\hline HMGB1 & 5'-GCACTCCCTCCATCTTTGGA-3' & 5'-CAGCTCCGACAGATCCAGTTC-3' \\
Mcl-1 & 5'-TGCTTCGGAAACTGGACATCA-3' & 5'-TAGCCACAAAGGCACCAAAAG-3' \\
-actin & 5'-CACTGTGTTGGCGTACAGGT-3' & 5'-TCATCACCATTGGCAATGAG-3' \\
\hline
\end{tabular}

$0.1 \%$ SDS, $0.1 \%$ sodium deoxycholate, and $1 \%$ NP40) containing protease and phosphatase inhibitors, and stored at $-20^{\circ} \mathrm{C}$. Samples $(30-50 \mu \mathrm{g})$ were separated by SDS-polyacrylamide gel electrophoresis and transferred to polyvinylidene difluoride (PVDF) membranes. Membranes were blocked with 5\% non-fat dry milk in Tris-buffered saline containing $0.05 \%$ Tween-20 (TBST) for $1 \mathrm{~h}$ at room temperature, then incubated with the following specific antibodies: rabbit anti-HMGB1, rabbit anti-Mcl-1 (Epitomics, Burlingame, CA, USA), or anti- $\beta$-actin (Cell Signaling Technology, New England BioLabs Inc., USA) for $1 \mathrm{~h}$ or overnight at $4^{\circ} \mathrm{C}$. After washing with TBST 3 times, membranes were incubated with secondary antibodies (horseradish peroxidase-conjugated anti-rabbit immunoglobulin; Santa Cruz Biotechnology) for $1 \mathrm{~h}$ at room temperature. Protein bands were visualized using an Anmobilon Western Chemiluminescent HRP Substrate system (Millipore Corp., Billerica, MA, USA).

Dual luciferase activity assay. The 3'-UTRs of HMGB1 and Mcl-1 mRNAs were PCR-amplified from human genomic DNA and inserted into the SpeI and HindIII sites in the pMIR-Report vector (Ambion Inc., Austin, TX, USA) downstream from the firefly luciferase coding sequence. 293T cells were cotransfected with pMIR-Report constructs, $m i R-181 b$ mimic or scramble control in combination with pRL-TK (Promega) using Lipofectamine 2000. Firefly and Renilla luciferase activities were determined using the dual luciferase reporter assay system (Promega) according to the manufacturer's instructions.

Statistical analysis. Data are expressed as means \pm standard errors of at least 3 independent experiments. Student's t-test and one-way analysis of variance were used to determine significance between groups. Statistical analysis was carried out using SPSS software (version 17.0). Differences with P-values of $<0.05$ were considered statistically significant.

\section{Results}

MiRNA expression in bone marrow blasts from AML patients and cell lines. To investigate whether miRNA participated in drug resistance in AML, we analyzed the expression of 3 miRNAs ( $m i R-181 b$, $m i R-451$ and $m i R-486$ ) in bone marrow samples from AML patients. The results showed that $m i R-181 \mathrm{~b}$ was downregulated in AML samples from relapsed/refractory patients in comparison with those of newly diagnosed AML 
A

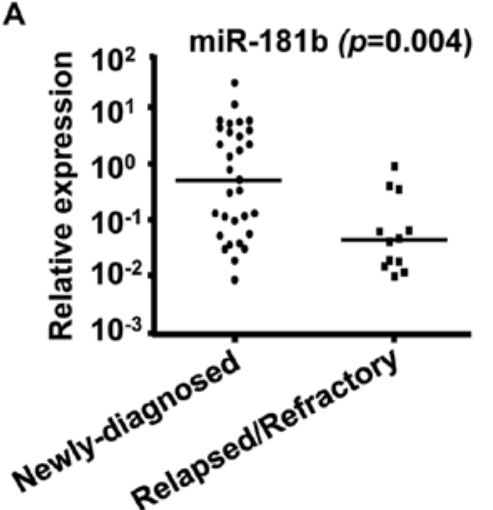

D

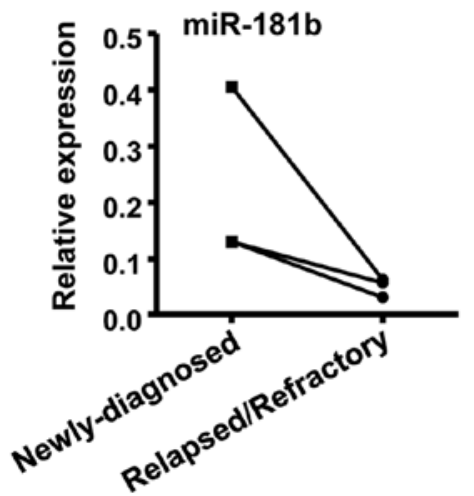

B

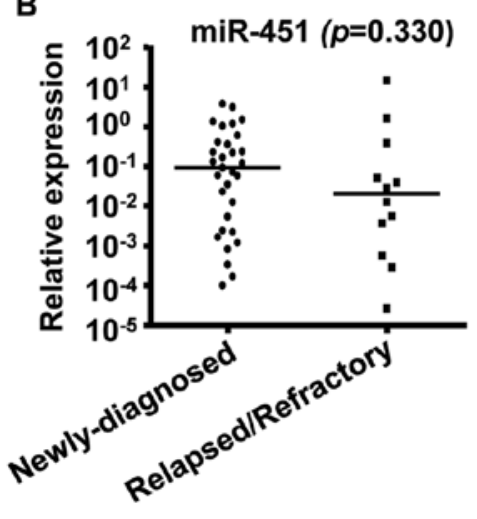

E

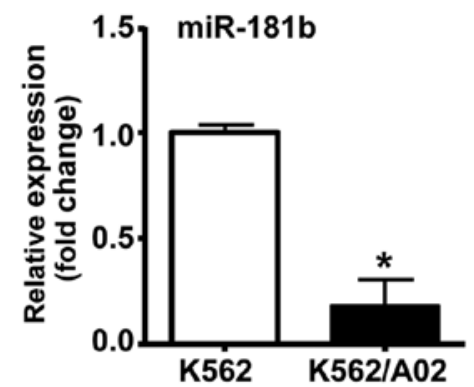

C

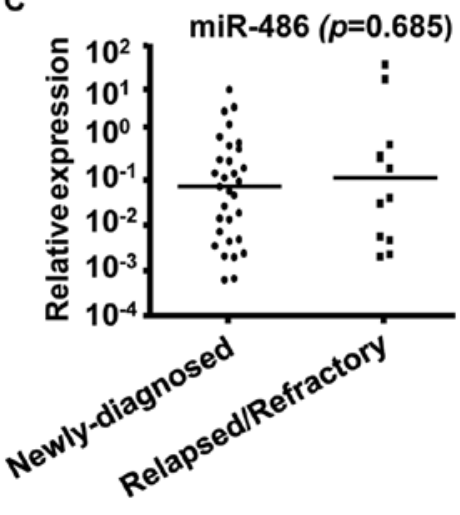

$\mathrm{F}$

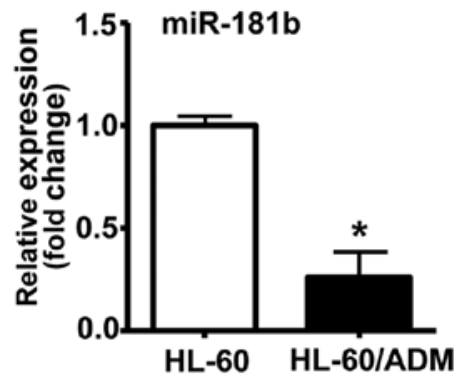

Figure 1. miRNA expression in bone marrow blasts from AML patients and AML cell lines. Expression levels of $m i R-181 b$ (A), $m i R-451$ (B) and $m i R-486$ (C) were determined by real-time PCR of bone marrow mononuclear cells (BM MNCs) from 31 patients with newly diagnosed AML and 12 patients with relapsed/ refractory AML. Solid points indicate individual values and horizontal lines represent medians. (D) Real-time PCR analysis of the $m i R$ - $181 b$ mRNA level in the same BM samples obtained at both the newly diagnosed and relapsed/refractory state. (E and F) $m i R$ - $181 b$ levels were analyzed by real-time PCR. Data are shown as fold changes of $m i R-181 b$ levels in K562/A02 and HL-60/ADM cells relative to K562 and HL-60 cells, respectively. "P<0.05.

patients (Fig. 1A, P<0.01). However, qRT-PCR assay revealed that there were no differences in $m i R-451$ or $m i R-486$ levels between the 2 groups of AML patients (Fig. 1B and C). BM samples from 3 paired AML patients were analyzed for $m i R-181 \mathrm{~b}$ levels at both the newly diagnosed and relapsed/ refractory state. We found a high level of $m i R-181 \mathrm{~b}$ at diagnosis, whereas a significant decrease in $m i R-181 b$ expression in the relapsed/refractory state (Fig. 1D).

Next, we performed quantitative RT-PCR to compare $m i R-181 b$ expression between human MDR leukemia cells and their parental drug-sensitive cells. As shown in Fig. 1E and F, the expression of $m i R-181 b$ was decreased significantly in human MDR leukemia cell lines K562/A02 and HL-60/ADM, as compared to their parental cell lines K562 and HL-60, respectively. These results suggested that $m i R-181 \mathrm{~b}$ may be involved in the development of drug resistance and disease progression in AML.

The levels of $m i R-181 b$ in newly diagnosed AML patients were then split into two classes (high and low expressions, according to the median expression in all samples). $m i R-181 \mathrm{~b}$ expression showed a negative correlation with treatment response in our enrolled cases, in which low expression of $m i R-181 b$ was observed more frequently in poor prognosis subset $(8 / 11,72.7 \%)$ than in good prognosis subset $(7 / 20,35 \%)$. These results confirm that low $m i R-181 b$ expression can act as a prognostic factor associated with poor outcome of AML patients.
Forced miR-181b expression sensitizes K562/A02 and HL-60/ ADM cells to chemotherapeutic agents. To further explore the effects of $m i R-181 \mathrm{~b}$ on chemoresistance in AML, we transiently transfected K562/A02 and HL-60/ADM cells with $m i R-181 b$ mimic or a negative control. Quantitative RT-PCR confirmed that $m i R-181 b$ mimic effectively enhanced the expression of miR-181b (Fig. 2A). Following transfection of drug-resistant cells with the mimic of $m i R-181 \mathrm{~b}$, we treated the cells with a series of concentrations of DOX or Ara-C for $48 \mathrm{~h}$. As shown in Fig. 2B, transfection with the miR-181b mimic significantly inhibited cell growth compared to transfection with the negative control. We next analyzed the effects of $m i R-181 b$ on apoptosis in AML by flow cytometry. The results showed that ectopic expression of $m i R-181 b$ markedly increased chemotherapy-inducing apoptosis (as measured by the percentage of Annexin V-FITC-positive cells) in AML drug-resistant cells (Fig. 2C-F). Taken together, these data indicated that the forced expression of $m i R-181 b$ increased the drug sensitivity of AML MDR cells to chemotherapy and promoted apoptosis.

$H M G B 1$ and Mcl-1 were identified as targets of miR-181b. The database Target Scan Human 6.2 was used to predict candidate targets of $m i R-181 b$. We identified HMGB1 and Mcl-1 as potential targets of $m i R-181 b$; these targets contain putative binding sites in the 3'-UTR that match with the 'seed' sequence of $m i R-181 b$ (Fig. 3A). To validate these interactions, 

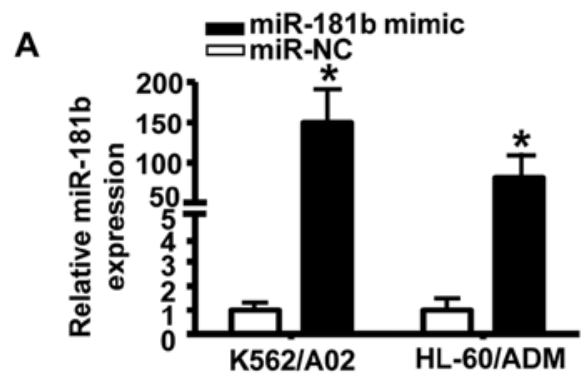

C

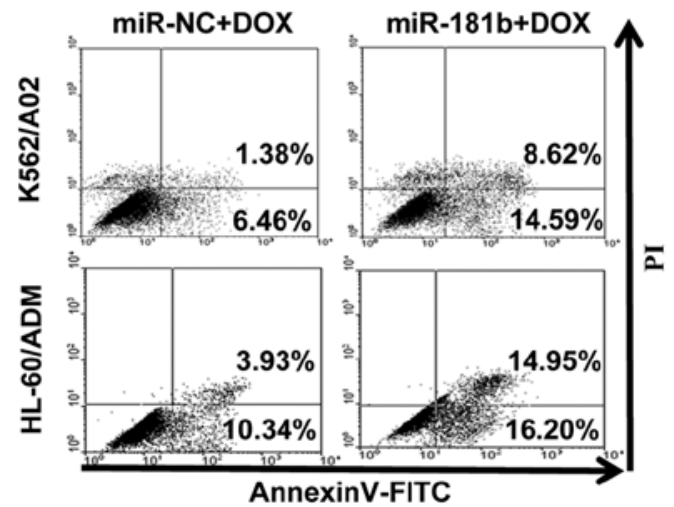

D

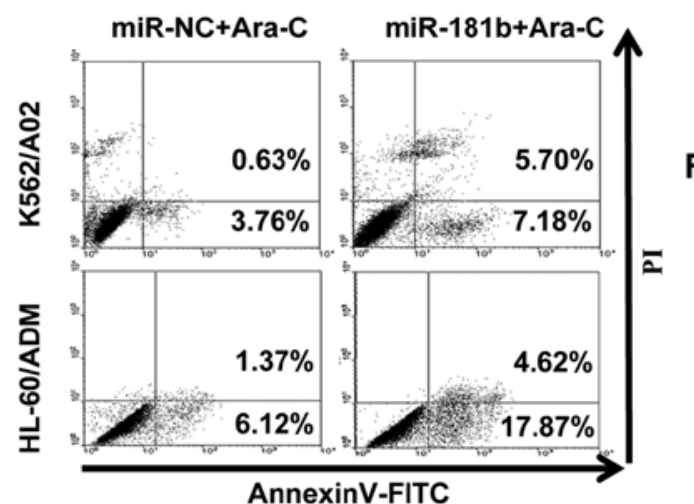

B
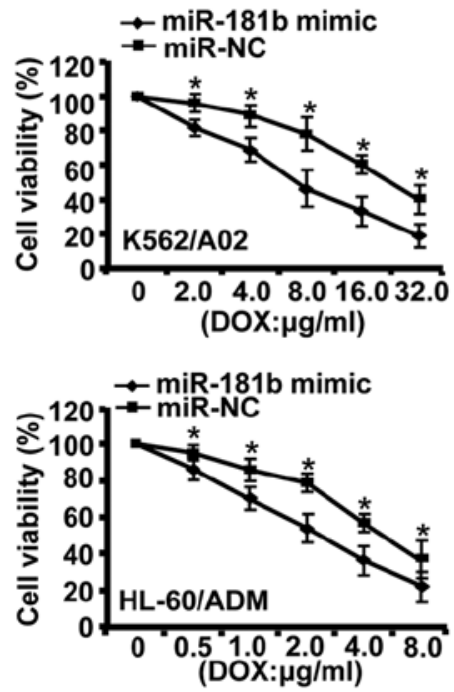

E

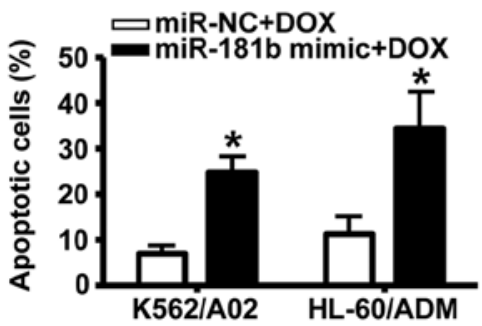

$\mathbf{F}$

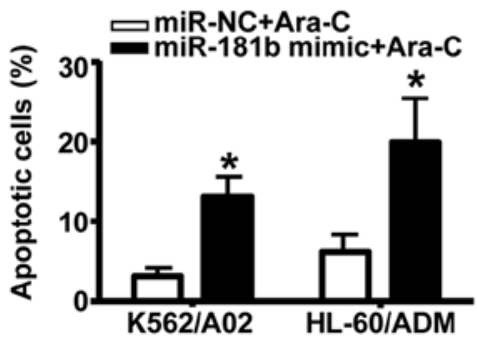

Figure 2. Forced expression of $m i R-181 b$ sensitized K562/A02 and HL-60/ADM cells to chemotherapeutic agents. (A) $m i R-181 b$ mimic (50 nM) was transfected into cells using Lipofectamine 2000. miR-NC was used as a control. Expression levels of $m i R-181 b$ were detected by real-time PCR. U6 snRNA was used as an internal control. Data are expressed as the mean \pm SD from at least 3 separate experiments. "P<0.05. (B) At $24 \mathrm{~h}$ after transfection, K562/A02 and HL-60/ADM cells were treated with serial dilutions of DOX or Ara-C for $48 \mathrm{~h}$. Cell viability was determined using a CCK8 assay. The $\mathrm{x}$-axis indicates the drug concentration of DOX or Ara-C. The error bar shows the standard deviation for 3 independent experiments. (C and D) Apoptosis in K562/A02 and HL-60/ADM cells was analyzed by flow cytometry using an Annexin V/PI detection kit after transfection with miR-181b or NC in the presence of DOX (C) or Ara-C (D) for $48 \mathrm{~h}$. A representative experiment is shown. (E and F) Percent of total apoptotic cells by quantitative analysis. "P<0.05.

we constructed luciferase reporter vectors carrying wild-type or mutated HMGB1 or Mcl-1 3'-UTR target sites and cotransfected these vectors with the $m i R-181 b$ mimic into $293 \mathrm{~T}$ cells. As illustrated in Fig. 3B, transfection with the $m i R-181 b$ mimic significantly decreases luciferase activity, whereas mutation of the 3'-UTR binding sites of HMGB1 or Mcl-1 in the reporter vector abrogated this effect, indicating that $m i R-181 b$ directly interacted with the 3'-UTR of HMGB1 and Mcl-1.

In order to verify whether $m i R-181 b$ affected endogenous levels of HMGB1 and Mcl-1 in AML, we analyzed HMGB1 and Mcl-1 expression after transfection with the $m i R-181 b$ mimic or inhibitor for $48 \mathrm{~h}$. The results revealed that the ectopic expression of $m i R-181 b$ in K562/A02 and HL-60/ ADM cells robustly suppressed endogenous HMGB1 and Mcl-1 expression both at mRNA and protein levels (Fig. 3C). Conversely, knockdown of $m i R-181 b$ by $m i R-181 b$ inhibitor markedly increased the expression of both HMGB1 and Mcl-1
(Fig. 3D). These results demonstrated that HMGB1 and Mcl-1 were direct targets of $m i R-181 b$ in AML MDR cells.

Restoration of miR-181b increased the drug sensitivity of AML MDR cells by targeting HMGB1 and Mcl-1. To further elucidate the role of HMGB1 in drug resistance in AML, we transfected K562/A02 and HL-60/ADM cells with HMGB1 siRNA; the effectiveness of the siRNAs designed to silence HMGB1 in cells is shown in Fig. 4A and B. Compared with negative controls, knockdown of HMGB1 dramatically decreased survival of K562/A02 and HL-60/ADM cells exposed to different concentrations of DOX or Ara-C (Fig. 4C). Annexin V/PI analysis showed that the proportion of apoptotic cells was significantly higher in HMGB1 siRNA-transfected cells compared to cells transfected with negative control siRNA (Fig. 4D-G). We previously reported that downregulation of Mcl-1 via RNA interference sensitized MDR leukemia 

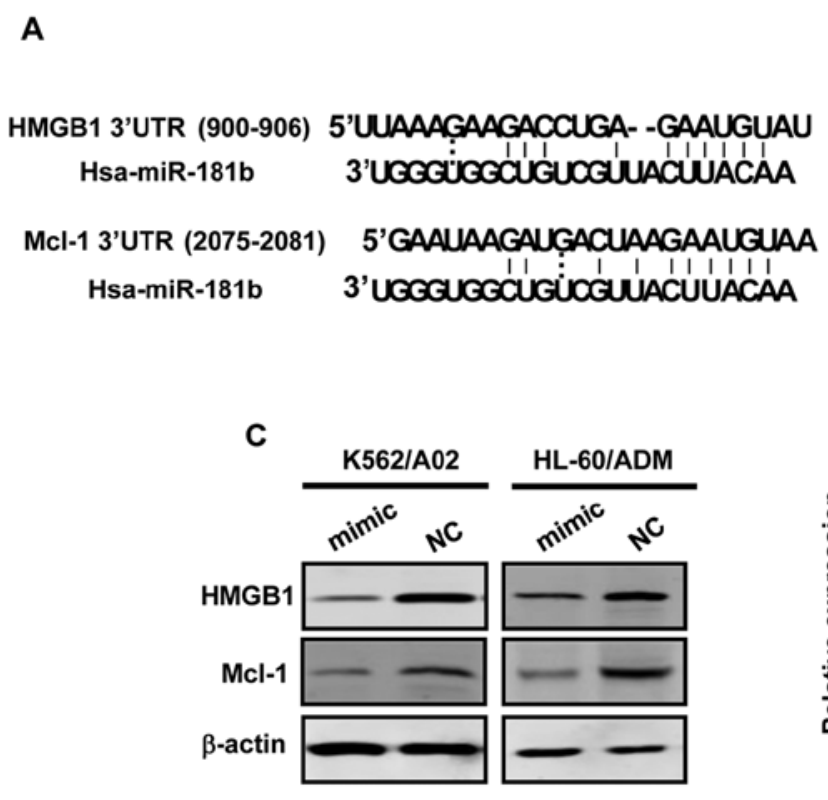

D

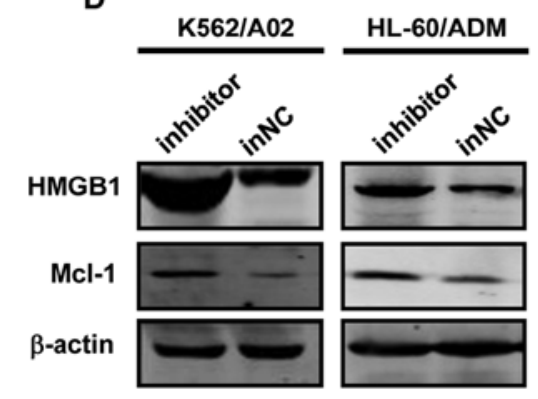

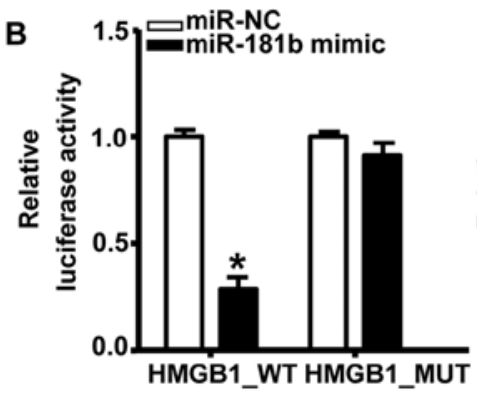
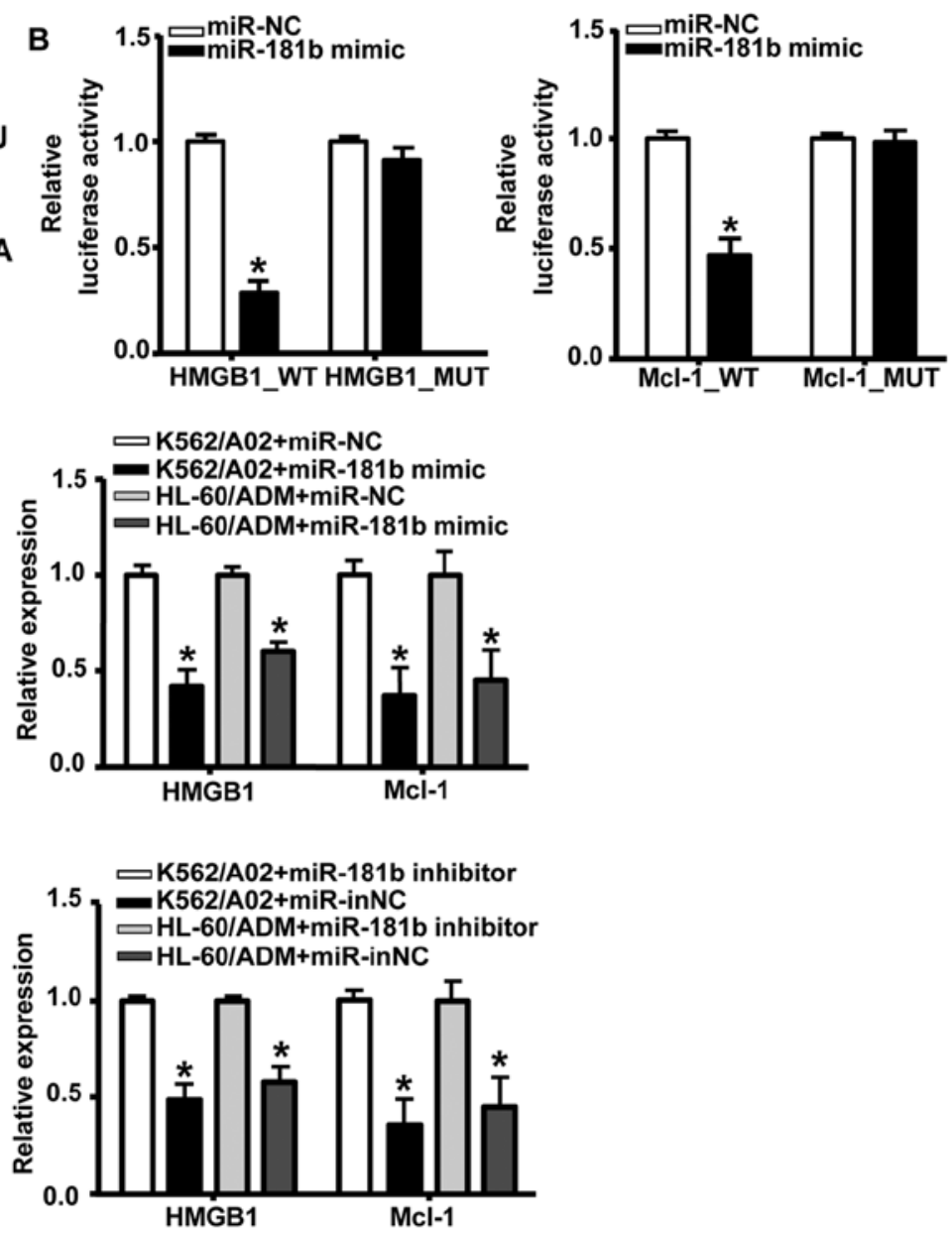

Figure 3. HMGB1 and Mcl-1 were identified as targets of $m i R-181 b$. (A) Predicted $m i R-181 b$ binding sites in HMGB1 and Mcl-1 3'-UTRs are shown. (B) A dual luciferase assay performed in 293T cells cotransfected with HMGB1_WT, HMGB1_MUT, Mcl-1_WT or Mcl-1_MUT report construct and either $m i R-181 \mathrm{~b}$ mimic or a scrambled control. Luciferase activities were calculated as a ratio of firefly to renilla luciferase activity and are expressed as means \pm SDs of 3 independent experiments. ${ }^{*}<0.05$. (C) Western blot (left) and real-time PCR (right) analysis of HMGB1 and Mcl-1 expression in K562/A02 and HL-60/ADM cells transfected with miR-181b mimic or miR-mimic negative control. " $\mathrm{P}<0.05$. (D) Western blot (left) and real-time PCR (right) analysis of HMGB1 and Mcl-1 expression in K562/A02 and HL-60/ADM cells transfected with miR-181b inhibitor or miR-inhibitor negative control. "P<0.05.

cells to chemotherapy and induced apoptosis (24). Thus, our results suggested that downregulation of Mcl-1 and HMGB1 was one pathway through which $m i R-181 b$ increased drug sensitivity in AML MDR cells.

Overexpression of $H M G B 1$ in relapsed/refractory $A M L$ patients. After verifying that HMGB1 was a target of $m i R-181 b$, we then sought to elucidate its role in AML. We first investigated HMGB1 expression by quantitative RT-PCR in BM cells obtained from 31 newly diagnosed AML patients and 12 patients with relapsed/refractory leukemia. As shown in Fig. 5A, HMGB1 expression was significantly increased in relapsed/refractory AML patients compared to newly diagnosed AML patients. Consistent with the real-time RT-PCR data, western blot analysis showed that HMGB1 protein levels were upregulated in relapsed/refractory AML patients than in newly diagnosed AML patients (Fig. 5B). We also noted an increase in the expression level of HMGB1 in sequential samples obtained from 3 paired AML patients and found that these HMGB1 levels were inversely correlated with $m i R-181 b$ expression levels (Fig. 5C). In conclusion, our data supported that the HMGB1 gene was aberrantly expressed in AML and was required for the development and progression of multidug resistance in AML.

\section{Discussion}

Expression and function analyses have unraveled the close relationship between aberrant $m i R-181 b$ expression and the pathogenesis, diagnosis, and prognosis of AML. It has been demonstrated that expression of $m i R-181 \mathrm{~b}$ is associated with lower CR rates and shorter relapse-free survival (RFS) and OS in adult patients with de novo AML (25). Multivariable analysis has revealed that increased expression of $m i R-181 a$ and $m i R-181 b$ is also significantly associated with favorable outcomes in cytogenetically abnormal AML with CEBPA mutations and cytogenetically normal AML patients (26-29). However, an obvious increase of $m i R-181 b-5 p$ was observed in AML serum samples and that higher expression levels of $m i R-181 b-5 p$ in serum are correlated with a poorer OS (30). Possible explanation for the different roles of $m i R-181 \mathrm{~b}$ in serum and tissues of AML patients could be the different origins and 
A

C
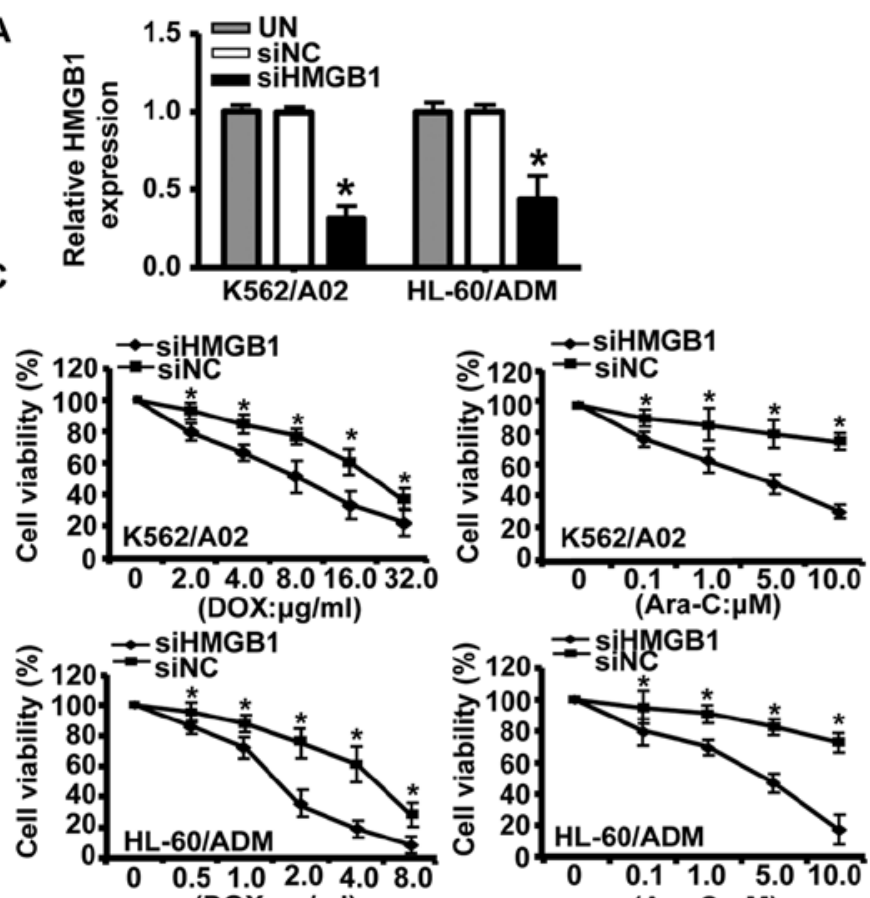

(DOX: $\mu \mathrm{g} / \mathrm{ml})$

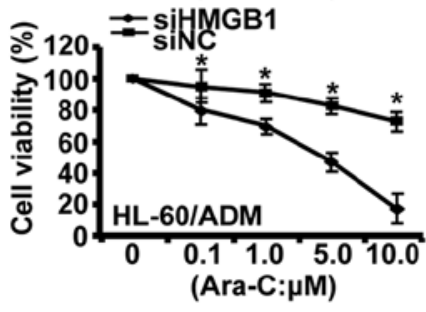

$F$

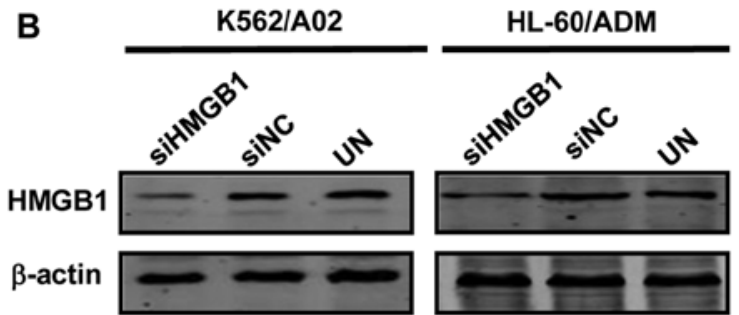

D
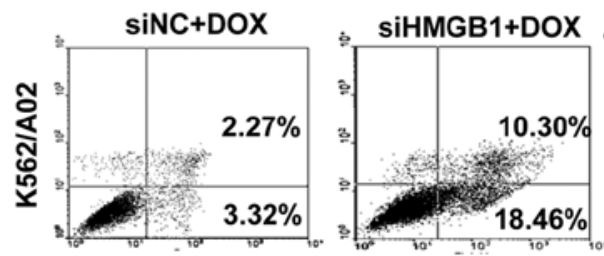

$\bar{\alpha}$

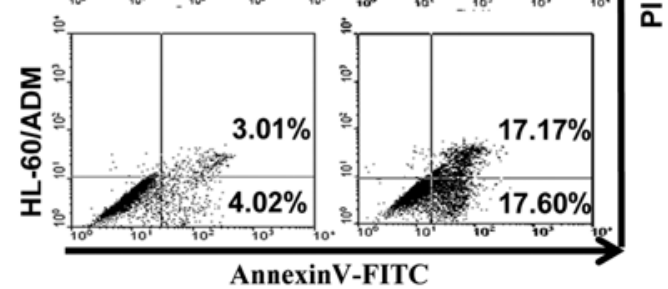

G
E

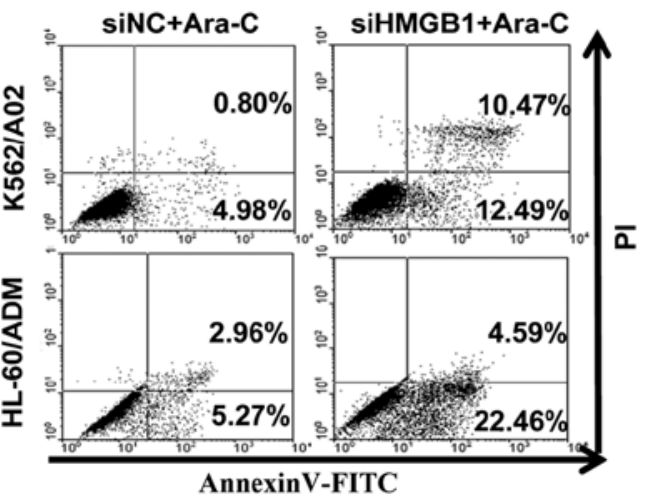

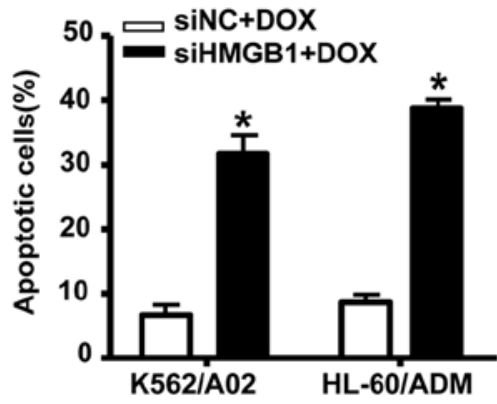

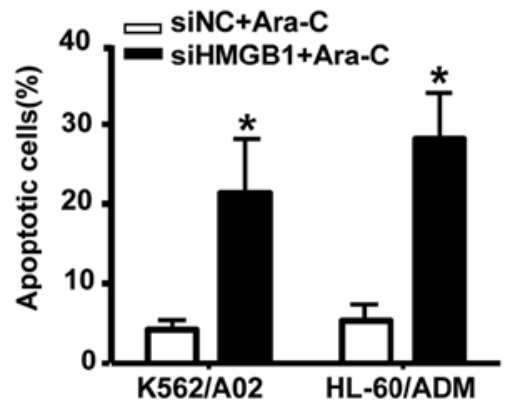

Figure 4. Role of HMGB1 in chemotherapy-resistant AML. K562/A02 and HL-60/ADM cells were untransfected (UN) or transiently transfected with HMGB1 siRNA (siHMGB1) or siRNA negative control (siNC) for $24 \mathrm{~h}$, and then the expression of HMGB1 was analyzed by real-time PCR (A) and western blot analysis (B). (C) CCK8 assays were used to detect the viability of K562/A02 and HL-60/ADM cells after incubating with DOX or Ara-C for $48 \mathrm{~h}$. The $\mathrm{x}$-axis indicates the drug concentration of DOX or Ara-C. Data plotted are the mean \pm SD of 3 separate experiments. (D and E) Annexin V/PI assays in K562/A02 and HL-60/ADM cells after transfection with HMGB1 siRNA or negative controls in the presence of DOX (D) or Ara-C (E) for $48 \mathrm{~h}$. A representative experiment is shown. ( $F$ and $\mathrm{G}$ ) Percent of total apoptotic cells by quantitative analysis. Columns indicate means of triplicate determinations and bars represent SDs. ${ }^{*} \mathrm{P}<0.05$.

AML patient samples used. These results also indicated that $m i R-181 b$ may be controlled by complex regulatory pathways in AML. miR-181a, another important member of the miR-181 family, was downregulated in the chemoresistant leukemia cell lines K562/A02 and HL-60/Ara-C compared to the parental K562 and HL-60 cells, and restoration of miR-181a expression could sensitize K562/A02 and HL-60/Ara-C cell lines to chemotherapeutic agents by targeting Bcl-2 $(31,32)$. However, the role of $m i R-181 \mathrm{~b}$ in the development of chemoresistance in AML cells is still unknown. The expression data reported in this study showed that among the selected miRNAs, only $m i R-181 b$ was differentially expressed in relapsed/refractory AML patients and newly diagnosed AML patients. Consistent with the results in AML patient samples, $m i R-181$ expression was lower in drug-resistant versus parental drug-sensitive AML cell lines. Additionally, in BM samples collected from 3 patients both at the diagnosis, prior to treatment and after relapse, we also noted decreases in the expression levels of $m i R-181 b$ in sequential samples. Although this cutoff point needs to be validated in an extended patient cohort, the current results suggested that lower expression of $m i R-181 b$ contributed to disease aggressiveness in AML. Furthermore, we verified that both AML drug-resistant cell lines K562/A02 and HL-60/ADM exhibited greatly enhanced sensitivity to DOX or Ara-C after transfection with the miR-181b mimic. These results suggested that $m i R-181 b$ may play an important role in the development and maintenance of MDR in AML.

HMGB1, a highly conserved DNA-binding protein, is ubiquitously expressed in the nuclei and cytoplasm of almost all eukaryotic cells. Within the nucleus, HMGB1 stabilizes nucleosome formation, assists in DNA mismatch repair, replication, and recombination, and regulates the transcription of many genes. Extracellular HMGB1 was identified as a prototypical damage-associated molecular pattern molecule 
A

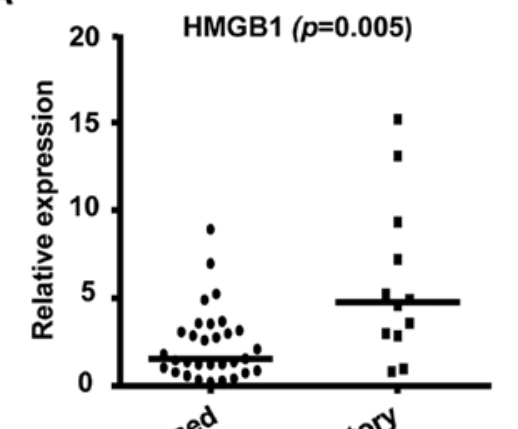

B

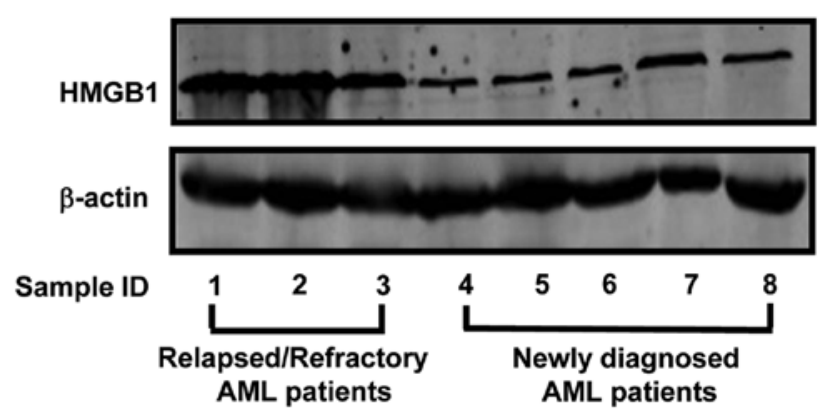

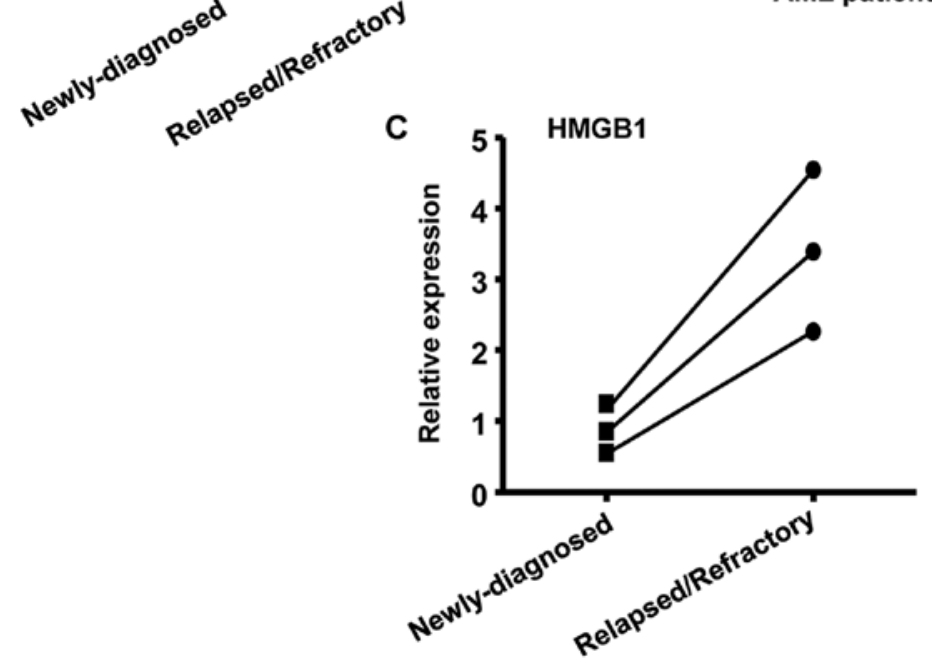

Figure 5. Overexpression of HMGB1 in relapsed/refractory AML patients. (A) Real-time PCR of HMGB1 in 31 newly diagnosed AML patients and 12 relapsed/ refractory AML patients. Solid points indicate individual values and horizontal lines represent medians. (B) Western blot analysis of HMGB1 in newly diagnosed AML patients and relapsed/refractory AML patients, $\beta$-actin was used as loading control. (C) Real-time PCR analysis of the HMGB1 mRNA level in the same BM samples obtained at both the newly diagnosed and relapsed/refractory state. ${ }^{*} \mathrm{P}<0.05$.

(DAMP) that is released both actively and passively from cells in response to infection or injury. Once released, HMGB1 can act as a chemokine or cytokine by ligation with specific receptors, including the receptor for advanced glycation end products (RAGE) and toll-like receptors (TLRs)-2, -4 and -9 (33). Recent studies demonstrated that the high expression of HMGB1 is tightly associated with unlimited replicative potential, angiogenesis, apoptosis, inflammation, invasion, and metastasis in cancer (34). Serum levels of HMGB1 are significantly higher in children with acute lymphoid leukemia (ALL) in the initial treatment group compared with healthy controls and the complete remission group (35). In addition to being involved in pathogenesis of leukemia, HMGB1 can be released from leukemia cell lines after chemotherapy-induced cytotoxicity and can promote chemotherapy resistance by inducing autophagy in leukemia cells (36). In this study, we showed that HMGB1 expression was significantly increased in relapsed/refractory AML patients compared to newly diagnosed patients. Inhibition of HMGB1 using siRNA enhanced drug sensitivity in leukemia cells, and this result was consistent with that in a previous study by Xie et al (37). Our study also identified HMGB1 as a direct and functional target of $m i R-181 b$. In addition, an obvious increase in HMGB1 levels and an inverse correlation with $m i R-181 \mathrm{~b}$ expression were also observed in blasts from the same AML patient. Thus, based on these results, HMGB1 appears to constitute a novel, powerful therapeutic target for AML patients.
Another well-distinguished target of $m i R-181 b$ in our study was Mcl-1, an anti-apoptotic member of the Bcl-2 family. Mcl-1 contains 3 BH domains and has a very short half-life. Functionally, Mcl-1 acts at mitochondria by binding to and sequestering a subset of $\mathrm{BH} 3$-only pro-apoptotic $\mathrm{Bcl}-2$ family members, including Bak, Bax, Bim, Bid, Bik, Noxa and Puma, thereby preventing the release of cytochrome $\mathrm{c}$ into the cytoplasm (38). The high expression of Mcl-1 in a wide variety of cancers is being intensively studied. Indeed, numerous reports have documented that overexpression of Mcl-1 protects cancer cells from apoptosis, representing a significant barrier to the efficacy of chemotherapeutic agents (39). Additionally, elevated expression of Mcl-1 was shown to correlate with leukemic relapse in AML patients (40), and recent studies have shown that Mcl-1 is upregulated in FMS-like tyrosine kinase-3-internal tandem duplication (FLT3-ITD)-positive AML cell lines and primary MNCs from AML patients. Mcl-1 is an essential effector of FLT3-ITD-mediated drug resistance, and suppression of endogenous Mcl-1 sensitizes FLT3-ITD-positive leukemias to cytotoxic therapies (41). We have previously reported that newly diagnosed or relapsed/ refractory leukemia patients express higher Mcl-1 levels than patients that are in complete remission. Consistent with this, knockdown of Mcl-1 sensitizes MDR leukemia cells to chemotherapy and induces apoptosis (24). In the present study, we demonstrated that $m i R-181 \mathrm{~b}$ directly regulated Mcl-1 expression post-transcriptionally in AML drug-resistant cell 
lines, suggesting that downregulation of Mcl-1 is one of the major mechanisms through which miR-181b promoted drug sensitivity in AML MDR cells. In CLL, Mcl-1 has also been identified as a target of $m i R-181 b$ and $m i R-181 a$, and increased Mcl-1 protein levels have been shown to be inversely correlated with decreased $m i R-181 b$ and miR-181a expression $(15,42)$. It is generally accepted that resistance to apoptosis is the main mechanism of drug resistance. The mitochondrial apoptotic pathway is tightly regulated by the Bcl-2 family. Suppression of HMGB1 by siRNA in K562/A02 leukemia cells promotes ADM-induced Smac/DIABLO release from the mitochondria to the cytoplasm, increasing the activation of caspase-3 (37). In addition, a recent study showed that autophagy-mediated HMGB1 release antagonizes vincristine-induced apoptosis in gastric cancer cells via transcriptional regulation of Mcl-1 and that HMGB1-mediated upregulation of Mcl-1 transcription is dependent on RAGE (43). Further in-depth studies are needed to investigate the interactions of HMGB1 and Mcl-1 in the regulation of AML drug resistance.

In conclusion, the present study showed that $m i R-181 b$ functioned as a tumor suppressor in AML chemoresistance. The abnormally decreased expression of $m i R-181 b$ was responsible for the occurrence of drug resistance in some AML patients. Forced expression of $m i R-181 b$ could enhance drug sensitivity and apoptosis in AML MDR cells at least partially though direct suppression of its target genes, HMGB1 and Mcl-1. Because the biological effects and regulatory networks of $m i R-181 b$ in AML are more complex than was once recognized, further studies are needed to confirm these results in an extended patient cohort. However, our data implied that ectopic implantation of $m i R-181 b$ alone or in conjunction with other anticancer agents may be a promising strategy to combat MDR in AML.

\section{Acknowledgements}

This study was supported by grants from the National Natural Science Foundation of China (81070422, 30871088, 81070407, 81000223), SRFDP of Educational Ministry (20100131110060), Medical and Health Science Technology Development of Shandong Province, China (2013WS0229).

\section{References}

1. Estey EH: Treatment of relapsed and refractory acute myelogenous leukemia. Leukemia 14: 476-479, 2000.

2. Chen Y, Tang Y, Guo C, Wang J, Boral D and Nie D: Nuclear receptors in the multidrug resistance through the regulation of drug-metabolizing enzymes and drug transporters. Biochem Pharmacol 83: 1112-1126, 2012.

3. Bartel DP: MicroRNAs: target recognition and regulatory functions. Cell 136: 215-233, 2009.

4. Garzon R, Marcucci G and Croce CM: Targeting microRNAs in cancer: rationale, strategies and challenges. Nat Rev Drug Discov 9: 775-789, 2010.

5. Nagano H, Tomimaru Y, Eguchi H, et al: MicroRNA-29a induces resistance to gemcitabine through the Wnt/beta-catenin signaling pathway in pancreatic cancer cells. Int J Oncol 43: 1066-1072, 2013.

6. Croce C: Introduction to the role of microRNAs in cancer diagnosis, prognosis, and treatment. Cancer J 18: 213-214, 2012 .

7. Feng DD, Zhang H, Zhang P, et al: Down-regulated miR-331-5p and miR-27a are associated with chemotherapy resistance and relapse in leukaemia. J Cell Mol Med 15: 2164-2175, 2011.
8. Li QJ, Chau J, Ebert PJ, et al: miR-181a is an intrinsic modulator of T cell sensitivity and selection. Cell 129: 147-161, 2007.

9. de Yebenes VG, Belver L, Pisano DG, et al: miR-181b negatively regulates activation-induced cytidine deaminase in B cells. J Exp Med 205: 2199-2206, 2008.

10. Wang B, Hsu SH, Majumder S, et al: TGFbeta-mediated upregulation of hepatic miR-181b promotes hepatocarcinogenesis by targeting TIMP3. Oncogene 29: 1787-1797, 2010.

11. Bisso A, Faleschini M, Zampa F, et al: Oncogenic miR-181a/b affect the DNA damage response in aggressive breast cancer. Cell Cycle 12: 1679-1687, 2013.

12. Lu Y, Roy S, Nuovo G, et al: Anti-microRNA-222 (anti-miR-222) and -181B suppress growth of tamoxifen-resistant xenografts in mouse by targeting TIMP3 protein and modulating mitogenic signal. J Biol Chem 286: 42292-42302, 2011.

13. Zhu W, Shan X, Wang T, Shu Y and Liu P: miR-181b modulates multidrug resistance by targeting BCL2 in human cancer cell lines. Int J Cancer 127: 2520-2529, 2010.

14. Marton S, Garcia MR, Robello C, et al: Small RNAs analysis in CLL reveals a deregulation of miRNA expression and novel miRNA candidates of putative relevance in CLL pathogenesis. Leukemia 22: 330-338, 2008.

15. Visone R, Veronese A, Rassenti LZ, et al: miR-181b is a biomarker of disease progression in chronic lymphocytic leukemia. Blood 118: 3072-3079, 2011.

16. Li S, Moffett HF, Lu J, et al: MicroRNA expression profiling identifies activated $\mathrm{B}$ cell status in chronic lymphocytic leukemia cells. PLoS One 6: e16956, 2011.

17. Visone R, Veronese A, Balatti V and Croce CM: MiR-181b: new perspective to evaluate disease progression in chronic lymphocytic leukemia. Oncotarget 3: 195-202, 2012.

18. Dore LC, Amigo JD, Dos Santos CO, et al: A GATA-1-regulated microRNA locus essential for erythropoiesis. Proc Natl Acad Sci USA 105: 3333-3338, 2008.

19. Wang R, Wang ZX, Yang JS, Pan X, De W and Chen LB: MicroRNA-451 functions as a tumor suppressor in human non-small cell lung cancer by targeting ras-related protein 14 (RAB14). Oncogene 30: 2644-2658, 2011.

20. Bian HB, Pan X, Yang JS, Wang ZX and De W: Upregulation of microRNA-451 increases cisplatin sensitivity of non-small cell lung cancer cell line (A549). J Exp Clin Cancer Res 30: 20, 2011.

21. Kovalchuk O, Filkowski J, Meservy J, et al: Involvement of microRNA-451 in resistance of the MCF-7 breast cancer cells to chemotherapeutic drug doxorubicin. Mol Cancer Ther 7: 2152-2159, 2008.

22. Wang J, Tian X, Han R, et al: Downregulation of miR-486-5p contributes to tumor progression and metastasis by targeting protumorigenic ARHGAP5 in lung cancer. Oncogene 33: 1181-1189, 2014.

23. Ragusa M, Majorana A, Statello L, et al: Specific alterations of microRNA transcriptome and global network structure in colorectal carcinoma after cetuximab treatment. Mol Cancer Ther 9: 3396-3409, 2010.

24. Ji M, Li J, Yu H, et al: Simultaneous targeting of MCL1 and $\mathrm{ABCB} 1$ as a novel strategy to overcome drug resistance in human leukaemia. Br J Haematol 145: 648-656, 2009.

25. Xiang L, Li M, Liu Y, et al: The clinical characteristics and prognostic significance of MN1 gene and MN1-associated microRNA expression in adult patients with de novo acute myeloid leukemia. Ann Hematol 92: 1063-1069, 2013.

26. Li Z, Huang H, Li Y, et al: Up-regulation of a HOXA-PBX3 homeobox-gene signature following down-regulation of miR-181 is associated with adverse prognosis in patients with cytogenetically abnormal AML. Blood 119: 2314-2324, 2012.

27. Garzon R, Garofalo M, Martelli MP, et al: Distinctive microRNA signature of acute myeloid leukemia bearing cytoplasmic mutated nucleophosmin. Proc Natl Acad Sci USA 105: 3945-3950, 2008.

28. Marcucci G, Maharry K, Radmacher MD, et al: Prognostic significance of, and gene and microRNA expression signatures associated with, CEBPA mutations in cytogenetically normal acute myeloid leukemia with high-risk molecular features: a Cancer and Leukemia Group B Study. J Clin Oncol 26: 5078-5087, 2008.

29. Marcucci G, Radmacher MD, Maharry K, et al: MicroRNA expression in cytogenetically normal acute myeloid leukemia. N Engl J Med 358: 1919-1928, 2008.

30. Zhi F, Cao X, Xie X, et al: Identification of circulating microRNAs as potential biomarkers for detecting acute myeloid leukemia. PLoS One 8: e56718, 2013. 
31. Li H, Hui L and Xu W: miR-181a sensitizes a multidrug-resistant leukemia cell line K562/A02 to daunorubicin by targeting BCL-2. Acta Biochim Biophys Sin (Shanghai) 44: 269-277, 2012.

32. Bai H, Cao Z, Deng C, Zhou L and Wang C: miR-181a sensitizes resistant leukaemia HL-60/Ara-C cells to Ara-C by inducing apoptosis. J Cancer Res Clin Oncol 138: 595-602, 2012.

33. Andersson U and Tracey KJ: HMGB1 is a therapeutic target for sterile inflammation and infection. Annu Rev Immunol 29: 139-162, 2011.

34. Tang D, Kang R, Zeh HJ III and Lotze MT: High-mobility group box 1 and cancer. Biochim Biophys Acta 1799: 131-140, 2010.

35. Kang R, Tang DL, Cao LZ, Yu Y, Zhang GY and Xiao XZ: High mobility group box 1 is increased in children with acute lymphocytic leukemia and stimulates the release of tumor necrosis factor-alpha in leukemic cell. Zhonghua Er Ke Za Zhi 45: 329-333, 2007 (In Chinese).

36. Liu L, Yang M, Kang R, et al: HMGB1-induced autophagy promotes chemotherapy resistance in leukemia cells. Leukemia 25: 23-31, 2011.

37. Xie M, Kang R, Yu Y, et al: Enhancive effect of HMGB1 gene silence on adriamycin-induced apoptosis in K562/A02 drug resistance leukemia cells. Zhonghua Xue Ye Xue Za Zhi 29: 549-552, 2008 (In Chinese).
38. Kozopas KM, Yang T, Buchan HL, Zhou P and Craig RW: MCL1, a gene expressed in programmed myeloid cell differentiation, has sequence similarity to BCL2. Proc Natl Acad Sci USA 90: 3516-3520, 1993.

39. Placzek WJ, Wei J, Kitada S, Zhai D, Reed JC and Pellecchia M: A survey of the anti-apoptotic Bcl-2 subfamily expression in cancer types provides a platform to predict the efficacy of Bcl-2 antagonists in cancer therapy. Cell Death Dis 1: e40, 2010.

40. Kaufmann SH, Karp JE, Svingen PA, et al: Elevated expression of the apoptotic regulator Mcl-1 at the time of leukemic relapse. Blood 91: 991-1000, 1998.

41. Kasper S, Breitenbuecher F, Heidel F, et al: Targeting MCL-1 sensitizes FLT3-ITD-positive leukemias to cytotoxic therapies. Blood Cancer J 2: e60, 2012.

42. Zhu DX, Zhu W, Fang C, et al: $\mathrm{miR}-181 \mathrm{a} / \mathrm{b}$ significantly enhances drug sensitivity in chronic lymphocytic leukemia cells via targeting multiple anti-apoptosis genes. Carcinogenesis 33: 1294-1301, 2012

43. Zhan Z, Li Q, Wu P, et al: Autophagy-mediated HMGB1 release antagonizes apoptosis of gastric cancer cells induced by vincristine via transcriptional regulation of Mcl-1. Autophagy 8: 109-121, 2012. 